\title{
LA MEJORA DEL RENDIMIENTO ESCOLAR Y EL CLIMA SOCIAL MEDIANTE UN PROGRAMA DE INTERVENCIÓN BASADO EN EL APRENDIZAJE-SERVICIO Y MENTORÍA ENTRE IGUALES
}

\section{IMPROVING THE SCHOOL PERFORMANCE AND SOCIAL CLIMATE THROUGH AN INTERVENTION PROGRAM BASED ON SERVICE-LEARNING AND PEER MENTORING}

\author{
Mirian Hervás Torres ${ }^{1}$ \\ Francisco D. Fernández Martín \\ José Luis Arco Tirado \\ Universidad de Granada, España \\ María Isabel Miñaca Laprida \\ Asociación Nuevo Futuro, España
}

\section{RESUMEN}

Los datos actuales, sobre fracaso escolar en educación son alarmantes, con índices por encima de la media europea Por ello, las universidades han de reaccionar con propuestas de mejora, fiables, válidas y adaptadas, tales como, el establecimiento de metodologías alternativas docentes, que ayudan al incremento de la adquisición de aprendizajes y competencias, las cuales reducen conductas de riesgo, promocionando un entorno y clima escolar adecuado. Atendiendo a esta situación, el objetivo de este estudio fue determinar la eficacia del Programa Huelva Educa, experiencia basada en el aprendizaje-servicio y la mentoría entre iguales, para incrementar el rendimiento escolar y mejorar el clima social de alumnado de educación obligatoria. La muestra estuvo compuesta por 69 alumnos de educación obligatoria y 14 docentes-tutores. En esta

${ }^{1}$ Correspondencia: Mirian Hervás Torres: Campus Universitario Cartuja s/n, Facultad de Ciencias de la Educación, Departamento de Psicología Evolutiva y de la Educación. Despacho no 134 (aulario). Universidad de Granada. C.P.: 18011, Granada (España). Correo-e: miriamhervas@ugr.es 
investigación, se empleó un diseño cuasiexperimental pretest-postest con un solo grupo, donde la intervención consistió en una serie de sesiones de mentoría entre alumnado universitario, previamente entrenado, y el alumnado de educación obligatoria, en horario extraescolar durante el curso escolar. Los resultados arrojan mejoras en el rendimiento escolar del alumnado de educación obligatoria, y mejoras en la cantidad y calidad de las interacciones en el aula, tanto desde la perspectiva del alumnado como de sus docentes-tutores, contribuyendo a la prevención del fracaso y abandono temprano.

Palabras clave: rendimiento escolar, clima escolar, aprendizaje-servicio, mentoría entre iguales, investigación evaluativa.

\section{ABSTRACT}

The current data about academic failure and dropout in education are alarming, with scores above average the European average. In this sense, the universities are to react with proposals, reliable, valid and adapted, such as, the establishment of teaching alternative methodologies, to increase the acquisition of learning and skills, reduces risk behaviors, and it promotes an environment and suitable school climate. Considering that situation, the aim of this study is to determine the efficacy of the Huelva Educa Program, an experience based on service-learning and peer mentoring, in order to increase school performance and improve the social climate in compulsory education students. The sample was composed of 69 students of compulsory education and 14 educational tutors. Likewise, in this investigation a quasi-experimental pretestposttest design with a single group was used, where the intervention, which consisted of different sessions of mentoring among university students, previously trained, and the pupils in compulsory education, in out of school hours during the scholastic year. The results show improvements in school performance of the pupils in compulsory education, and improvements in the quantity and quality of interactions in the classroom, both from the perspective of students and of the educational-tutors, contributing to the failure and early dropout prevention.

Key Words: school performance, social climate, service-learning, peer mentoring, evaluative research.

\section{Introducción}

La conceptualización de fracaso escolar es una tarea compleja, debido principalmente a su carácter polisémico y su uso en diferentes contextos (Lara, González, González, y Martínez, 2014). Este concepto, en ocasiones, hace referencia al alumnado que obtiene un bajo rendimiento escolar, en otras, alude al alumnado que abandona o finaliza la Educación Secundaria Obligatoria (ESO) sin la titulación correspondiente, aunque también se suele asociar al alumnado que no supera la Educación Secundaria Postobligatoria. No obstante, en un intento de unificación del término, podría definirse en los términos que indica Martínez (2009) como "toda insuficiencia detectada en los resultados alcanzados por los alumnos en los centros de enseñanza respecto a los objetivos propuestos para su nivel, edad y desarrollo, y que habitualmente se expresa a través de calificaciones escolares negativas" (p. 13).

Actualmente, el concepto de fracaso escolar se asocia en mayor medida a la etapa de ESO, aunque sus inicios se pueden situar en la etapa de Educación Primaria (EP) como indican los estudios de Fernández, Mena y Riviere (2010). En esta línea, los datos registrados en el curso escolar 2013-2014, en el conjunto del territorio nacional, el 2,3\% de la población de EP fueron 
repetidores, correspondiendo el 2,5\% a alumnado perteneciente al primer ciclo, el 2,2\% al segundo ciclo y el 2,4\% al tercer ciclo (Consejo Escolar del Estado, 2015). Asimismo, mencionar el bajo nivel en competencia básicas que se registraron en esta etapa (ej., comprensión lectora, matemáticas y ciencias), tal y como revelan los resultados de los Estudios Internacionales (Instituto Nacional de Evaluación Educativa, 2014).

Sin embargo, en la etapa de ESO el rendimiento en estos indicadores de resultados educativos se reduce considerablemente, ubicándose en el año 2014 la tasa de abandono temprano de la educación y la formación en un 21,9\% (Instituto Nacional de Evaluación Educativa, 2015), aunque reduciéndose esta cifra en 2015 en 1,9 puntos (20\%) (Instituto Nacional de Evaluación Educativa, 2016). Por otro lado, en el curso escolar 2013-2014, aunque hay un ligero ascenso en la tasa de idoneidad del alumnado de 12 años (84,9\%) y 15 años (63,6\%), el 15,1\% del alumnado de EP no inicia los estudios de secundaria a la edad adecuada y el $36,4 \%$ del alumnado de 15 años no está matriculado en el curso que le corresponde por su edad, sino en alguno anterior (Consejo Escolar del Estado, 2015). En este mismo curso escolar, a los 16 años, edad teórica de inicio de la etapa de educación secundaria postobligatoria (Bachillerato o enseñanzas profesionales de grado medio), el 31,1\% de la población cursaba todavía estudios de ESO y el 56,3\% restante estaba escolarizado en primer curso de la educación secundaria postobligatoria (el 52,4\% en Bachillerato, el 3,9\% en enseñanzas profesionales de grado medio y el 7,6\% estaba matriculado en un Programa de Cualificación Profesional Inicial), estimándose que la población de 16 años sin escolarizar alcanzaba un valor del 3,4\% (Consejo Escolar del Estado, 2015).

A estos alarmantes resultados de rendimiento se unen las deficiencias en competencias clave del alumnado de ESO, como reflejan los resultados globales en competencias en matemáticas, comprensión lectora, ciencias y resolución de problemas, con valores de 484, 488, 496 y 477 puntos, respectivamente (Instituto Nacional de Evaluación Educativa, 2014). Si bien se ha producido una leve mejora respecto a los estudios internacionales previos, la puntuación media obtenida por el alumnado español en estas competencias sigue ubicándose por debajo de las puntuaciones medias de referencia, por lo que se hace prioritario mejorar la calidad y eficacia de la educación y formación obligatoria española, atendiendo a factores como (Sammons y Bakkum, 2011): (a) la orientación del logro, (b) la calidad del currículo, (c) el clima social, (d) el grado de participación de los padres, (e) la cantidad de tiempo de aprendizaje efectivo, (f) la gestión del aula, (g) el grado de estructuración de las instrucciones, (h) el nivel de autonomía en el aprendizaje, e (i) el nivel de adaptación y retroalimentación.

Partiendo de los datos registrados por el alumnado de educación obligatoria, se hace necesario examinar la situación de las instituciones educativas, principalmente conocer el proceso de Enseñanza-Aprendizaje de los contenidos que conforman el currículum, prestando especial atención a las interacciones sociales en el aula o clima social, que éste proceso genera (Molina y Pérez, 2006), pues las relaciones interpersonales contribuyen al crecimiento, aprendizaje y mejora del desarrollo (Noam y Fiore, 2004) y tienen un efecto directamente proporcional al grado en el que el alumnado percibe que sus docentes les apoyan, con expectativas claras y justas frente a sus individualidades, muestra afectividad y les motivan (González, Rivera, y Trigueros, 2014).

Esta situación justifica la necesidad de buscar procedimientos que permitan aumentar los aprendizajes escolares, prevenir el fracaso y abandono escolar temprano, mejorar el clima social, etc., del alumnado de educación obligatoria, como es el caso del Programa Huelva Educa (PHE), fundamentado, por un lado, en la metodología alternativa de Aprendizaje-Servicio (A-S), entendida como una metodología donde los participantes desarrollan habilidades, destrezas, competencias, etc., realizando un servicio a su comunidad (Westover, 2012), y por otro lado, el aprendizaje entre iguales en su versión de mentoría, donde una persona con mayor experiencia ayuda a otra más joven en aspectos esenciales de su vida (académicos, personales, etc.) (Arco, Fernández, y Fernández, 2011). Aunque se podría haber optado por una mentoría tradicional, grupal de equipo o virtual, la elección de la mentoría entre iguales se debió, por un lado, por la oportunidad que se 
produce entre mentor y "mentee" al desarrollar una relación de orientación (académica y personal) y enseñanza con altos niveles de satisfacción y, por otro lado, porque ofrece al alumnado de educación obligatoria modelos de rol positivos, debido a la cercanía de edad entre ambos.

En este programa, alumnado de titulaciones relacionadas con la profesión docente de la Universidad de Huelva (UHU) (ej., Grado de Psicología, Grado de Educación Social, Grado de Educación Primaria, etc.), previo proceso de formación y entrenamiento, en calidad de mentores, a lo largo del segundo y tercer trimestre del curso escolar, llevan a cabo sesiones semanales de mentoría altamente estructuradas, de carácter individual y grupal, con alumnado de tercer ciclo de EP y primer ciclo de ESO, con el propósito de mejorar su rendimiento escolar, características socio-ambientales y relaciones personales en el aula.

Este tipo de programas presentan un alto nivel de eficacia y eficiencia, destacando entre sus resultados: (a) un aumento en el compromiso escolar del alumnado (Maloney y Griffith 2013; McIntyre y Sellnow 2014), (b) mejoras en el aprendizaje escolar (McIntyre y Sellnow, 2014; Vaknin y Bresciani, 2013), (c) el fomento de la reflexión y el pensamiento crítico entre el alumnado (Stanton, 2014; Vaknin y Bresciani, 2013), (d) la estimulación de aspectos relacionados con el desarrollo personal y social del alumnado (Hervás, Fernández, Arco, y Miñaca, 2017; Knapp, Fisher, y Levesque-Bristol, 2010; McIntyre y Sellnow, 2014; Risquez, 2011), (e) una mayor vinculación del alumnado a la institución educativa (Mclntyre y Sellnow, 2014; Wilczenski y Coomey, 2007), (f) una mejora en el clima social (Weiler, LaGoy, Crane, y Rovner, 1998), (g) un incremento de la motivación del profesorado, su satisfacción y actitud hacia la enseñanza (Lord et al., 2012; Wilczenski y Coomey, 2007), y (h) aumento del apoyo a la escuela por parte de la comunidad y mejora en la comunidad de su estructura organizativa y participación social de sus miembros (Maloney, Myers, y Bazyk, 2014; McIntyre y Sellnow, 2014; Westover, 2012).

En definitiva, el objetivo de este estudio fue conocer la eficacia del PHE para incrementar el rendimiento escolar del alumnado de educación obligatoria participante y mejorar el clima social desde la perspectiva del alumnado de educación obligatoria y los docentes-tutores participantes. Las hipótesis establecidas fueron: (1) el alumnado de educación obligatoria participante incrementará en términos estadísticamente significativos su rendimiento escolar en las diferentes áreas curriculares en la fase postest respecto a la fase pretest tras participar en el PHE, (2) existen diferencias estadísticamente significativas en las puntuaciones directas medias obtenidas por el alumnado de educación obligatoria participante en características socio-ambientales y relaciones personales en el aula en la fase postest respecto a la fase pretest como resultado de su participación en el programa, y (3) los docentes-tutores participantes mejorarán a nivel estadísticamente significativo sus puntuaciones directas medias logradas en características socioambientales y relaciones personales en el aula en la fase postest respecto a la fase pretest tras participar en el PHE.

\section{Método}

\section{Muestra}

La muestra de esta investigación estuvo compuesta por un total de 83 sujetos de 2 Centros de Educación Infantil y Primaria (CEIP) y 3 Institutos de Educación Secundaria (IES) de la ciudad de Huelva, divididos en 2 submuestras, por un lado, alumnado de educación obligatoria y, por otro lado, docentes-tutores de la submuestra de alumnado de educación obligatoria.

La submuestra de alumnado de educación obligatoria estuvo compuesta de 69 alumnos: 31 de EP (10 de $5^{\circ}$ curso y 21 de $6^{\circ}$ curso) y 38 de ESO (12 de $1^{\circ}$ curso y 26 de $2^{\circ}$ curso). Su media de edad era de 12,26 años (rango de entre 10 y 15 años), y por sexo, 30 eran mujeres y 39 
hombres. Por su parte, la submuestra de docentes-tutores se compuso de 14 docentes-tutores: 4 de EP ( 1 de $5^{\circ}$ curso y 3 de $6^{\circ}$ curso) y 10 de ESO ( 5 de $1^{\circ}$ curso y 5 de $2^{\circ}$ curso). Su media de edad era de 41,02 años (rango de entre 30 y 61 años). Por sexo, participaron 6 mujeres y 8 hombres (tabla 1).

TABLA 1. Información Escolar Alumnado de EP y ESO e Información demográfica docentes tutores

\begin{tabular}{l|ccccc|cc}
\hline \multirow{2}{*}{ Dato escolar } & \multicolumn{3}{|c}{ CEIP 1 } & \multicolumn{3}{c|}{ CEIP 2 } & \multicolumn{2}{c}{ Total } \\
\cline { 3 - 8 } & & $N$ & $\%$ & $N$ & $\%$ & $N$ & $\%$ \\
\hline Total & 5 & 14,17 & 26 & 76,47 & 31 & 44,92 \\
Curso & 50 & 0 & 0,00 & 10 & 38,46 & 10 & 32,25 \\
& 6o & 5 & 100 & 16 & 61,54 & 21 & 67,75 \\
\hline
\end{tabular}

\begin{tabular}{|c|c|c|c|c|c|c|c|c|c|}
\hline \multirow{2}{*}{ Dato escolar } & & \multicolumn{2}{|c|}{ IES 1} & \multicolumn{2}{|c|}{ IES 2} & \multicolumn{2}{|c|}{ IES 3} & \multicolumn{2}{|c|}{ Total } \\
\hline & & $N$ & $\%$ & $N$ & $\%$ & $N$ & $\%$ & $N$ & $\%$ \\
\hline Total & & 24 & 63,15 & 9 & 23,68 & 5 & 13,15 & 38 & 55,08 \\
\hline Curso & 10 & 7 & 29,17 & 1 & 11,11 & 4 & 80,00 & 12 & 31,67 \\
\hline & $2^{\circ}$ & 17 & 70,83 & 8 & 88,88 & 1 & 20,00 & 24 & 68,33 \\
\hline
\end{tabular}

\begin{tabular}{|c|c|c|c|c|c|c|c|c|c|c|c|c|}
\hline \multirow{3}{*}{$\begin{array}{l}\text { Dato } \\
\text { demográfico }\end{array}$} & \multicolumn{4}{|c|}{ Educación Primaria } & \multicolumn{6}{|c|}{ Educación Secundaria } & \multicolumn{2}{|c|}{ Total } \\
\hline & \multicolumn{2}{|c|}{ CEIP 1} & \multicolumn{2}{|c|}{ CEIP 2} & \multicolumn{2}{|c|}{ IES 1} & \multicolumn{2}{|r|}{ IES 2} & \multicolumn{2}{|r|}{ IES 3} & \multirow[b]{2}{*}{$N$} & \multirow[b]{2}{*}{$\%$} \\
\hline & $N$ & $\%$ & $N$ & $\%$ & $N$ & $\%$ & $N$ & $\%$ & $N$ & $\%$ & & \\
\hline $\begin{array}{l}\text { Total } \\
\text { Edad }\end{array}$ & 2 & 14,29 & 2 & 14,29 & 6 & 42,84 & 2 & 14,29 & 2 & 14,29 & 14 & 100 \\
\hline $\begin{array}{l}\text { Media } \\
\text { Rango }\end{array}$ & \multicolumn{2}{|c|}{$\begin{array}{c}33,5 \\
33-34\end{array}$} & \multicolumn{2}{|c|}{$\begin{array}{c}40,5 \\
40-41\end{array}$} & \multirow{2}{*}{\multicolumn{2}{|c|}{$\begin{array}{c}35,5 \\
30-61\end{array}$}} & \multirow{2}{*}{\multicolumn{2}{|c|}{$\begin{array}{c}46 \\
45-47\end{array}$}} & \multirow{2}{*}{\multicolumn{2}{|c|}{$\begin{array}{c}42,5 \\
37-48\end{array}$}} & \multirow{2}{*}{\multicolumn{2}{|c|}{$\begin{array}{l}57,14 \\
30-61\end{array}$}} \\
\hline Sexo & & & & & & & & & & & & \\
\hline Mujer & 2 & 100 & 1 & 50,00 & 3 & 50,00 & 1 & 50,00 & 1 & 50,00 & 8 & 57,15 \\
\hline Hombre & 0 & 0,00 & 1 & 50,00 & 3 & 50,00 & 1 & 50,00 & 1 & 50,00 & 6 & 42,85 \\
\hline
\end{tabular}

Fuente: Elaboración propia

El procedimiento de selección de la muestra se basó en un tipo de muestreo no probabilístico denominado "muestras de conveniencia" (Martínez, 2010): (a) primera etapa: la parte de la población a la que se tenía acceso correspondiente al alumnado de los diferentes CEIP e IES de la ciudad de Huelva, (b) segunda etapa: se seleccionaron aquellos CEIP e IES más próximos al Campus universitario "El Carmen" de la UHU, (c) tercera etapa: diferentes sesiones de divulgación del programa en los centros educativos seleccionados en la segunda etapa (ej., características y condiciones de participación en el programa), solicitando paralelamente su participación de manera voluntaria en el mismo, lo que generó la inscripción de 2 CEIP y 3 IES, que fueron los que aceptaron las condiciones de participación, y (d) cuarta etapa: selección de las submuestras, donde los docentes-tutores de los centros que finalmente fueron seleccionados, con el consentimiento de las familias, tramitaron la inscripción del alumnado de educación obligatoria $(N$ = 69) en el programa en diferentes momentos temporales, para lo que implementaron las siguientes actuaciones: (1) lectura de la Solicitud del centro para apoyo externo del alumnado de EP y ESO (documento cuyo objetivo fue establecer el compromiso, derechos y deberes de los centros educativos participantes) y firma del mismo, (2) completar el Protocolo para apoyo externo del alumnado de EP y ESO (documento donde se recogió información demográfica, escolar y de interés del alumnado de educación obligatoria), y (3) aportar la Solicitud de la familia para apoyo externo del alumnado de EP y ESO (documento cuyo propósito era establecer el compromiso, derechos y deberes de las familias del alumnado de educación obligatoria) firmada. Asimismo, la 
inscripción del alumnado en el programa, implicaba directamente la inscripción en el mismo de los docentes-tutores $(N=14)$.

\section{Variables}

Las variables para este estudio fueron:

1. Rendimiento escolar: término que hace referencia al nivel de conocimientos y destrezas escolares exhibidos por los estudiantes (Pulido y Herrera, 2016).

2. Clima social: entendido como las relaciones del alumnado-profesorado y profesoradoalumnado y la estructura organizativa de la clase (Moos, Moos, y Trickett, 1995).

\section{Instrumentos}

Boletín de calificaciones. Copia original del boletín de calificaciones donde se especifican los datos personales del alumnado de educación obligatoria, las calificaciones obtenidas en cada una de las áreas curriculares cursadas en las diferentes evaluaciones, aquellas materias que han sido objeto de adaptación curricular y/o que necesiten refuerzo educativo, la decisión de promoción, un resumen de faltas de asistencia y observaciones. Esta información se empleó para determinar el rendimiento escolar del alumnado de educación obligatoria en las diferentes evaluaciones del curso escolar.

Escala de Clima Social: Escolar (CES). Instrumento compuesto por 90 ítems con dos alternativas de respuesta (verdadero y falso), agrupados en 4 dimensiones con 9 subescalas: (a) dimensión relacional o relaciones, mide en qué medida los estudiantes están integrados en la clase, se apoyan y ayudan entre sí: Implicación, Afiliación y Ayuda, (b) dimensión de desarrollo personal o autorrealización, valora la importancia que se concede en la clase a la realización de las tareas y a los temas de las materias: Tarea y Competitividad, (c) dimensión de estabilidad o del sistema de mantenimiento, evalúa las actividades relacionadas con el cumplimiento de objetivos, funcionamiento adecuado de la clase, organización, claridad y coherencia: Organización, Claridad y Control, y (d) dimensión del sistema de cambio, evalúa el grado en que existe diversidad, novedad o variación de las actividades de clase: Innovación. Fue desarrollada inicialmente por Moos y Tricket (1979), y posteriormente por Moos et al. (1995), y se utilizó por ser una de las pruebas que generalmente se emplea para evaluar las características socioambientales y relaciones personales en el aula (ej., relaciones del alumnado con el profesorado y estructura organizativa en el aula) desde la perspectiva del alumnado de educación obligatoria, y debido a su satisfactoria fiabilidad (modelo test-retest: entre ,72 y ,90 puntos; y Kuder y Richardson 20-21: entre ,67 y ,86 puntos) y validez (análisis diferenciales y factoriales que justifican la estructura de la prueba).

\section{Aplicación del programa de intervención}

Una vez determinadas las submuestras de alumnado de educación obligatoria y docentestutores, y previa selección y entrenamiento en un seminario de formación al alumnado universitario que actuarían como mentores en el PHE (primera actividad del plan de intervención), se procedió al emparejamiento entre el alumnado de educación obligatoria, con su correspondiente mentor, considerando para ello la disponibilidad horaria y la equivalencia en experiencia de vida e intereses (Mentor/National Mentoring Partnership, 2005). En este momento, los responsables del programa aportaron a cada mentor información (ej., demográfica, escolar y de interés) del alumnado asignado, facilitándole igualmente los materiales necesarios para el desarrollo de las sesiones de mentoría (ej., Cuaderno de trabajo de mentores PHE, instrumento donde se estructuraron las sesiones de mentoría además de emplearse durante el plan de 
seguimiento del programa) y datos de contacto de la familia para establecer la fecha y horario de la primera sesión de mentoría.

Conformadas las parejas de mentores y alumnado de educación obligatoria, se iniciaron las sesiones de mentoría entre ambos (segunda actividad que conformaba el plan de intervención), desarrollándose en horario extraescolar a lo largo del segundo y tercer trimestre del curso escolar, tanto en formato individual (cada mentor con su respectivo alumno de educación obligatoria) como grupal (varios mentores con sus respectivos alumnos de educación obligatoria en actividades comunes).

La frecuencia de las sesiones de mentoría era semanal y con una duración de 90 minutos, realizándose en los horarios fijados previamente entre los implicados, y en los lugares habilitados por los responsables del programa, es decir, las aulas de los centros educativos de referencia o las aulas proporcionadas por la UHU. Las actividades llevadas a cabo en las diferentes sesiones de mentoría con el alumnado de educación obligatoria se adecuaron a sus necesidades, que fueron identificadas a partir de la información aportada por los docentes-tutores (Protocolo para apoyo externo del alumnado de EP y ESO), y la información recogida por los mentores en la primera sesión de mentoría, en los términos que recomienda la literatura especializada (Boland, 2010; Miñaca y Hervás, 2013), dirigiéndose al: (a) refuerzo escolar en áreas instrumentales: intervención en dificultades de aprendizaje, entrenamiento en estrategias de aprendizaje cognitivas, metacognitivas y de apoyo, entre otras, (b) actividades de orientación personal: gestión de emociones, habilidades sociales, etc., (c) actividades de orientación vocacional-profesional: transición de EP a ESO, itinerarios formativos, información sobre titulaciones, etc., y (d) actividades de gestión del ocio y tiempo libre: actividades grupales para una mejor socialización del alumnado en su grupo-clase, resolución de conflictos, entre otras.

En total, se efectuaron 955 sesiones de mentoría $\left(\bar{x}=13,84 ; \sigma_{x}=2,51\right.$; rango entre 9 y 18 sesiones), de las cuales 878 fueron de carácter individual ( $\bar{x}=12,72 ; \sigma_{X}=2,90$; rango entre 4 y 18 sesiones), y 77 de carácter grupal ( $\overline{\mathrm{X}}=1,11 ; \sigma_{\mathrm{X}}=1,66$; rango entre 0 y 10 sesiones). Si indicar que las diferencias en el número de sesiones de mentoría en las que participó el alumnado de educación obligatoria se deben a los diferentes momentos en los que dicho alumnado fue inscrito, así como a las sesiones de mentoría que fueron canceladas $(N=184)$ por diferentes motivos, tanto individuales $\left(N=132 ; \overline{\mathrm{x}}=1,91 ; \sigma_{\mathrm{X}}=, 78\right.$; rango entre 1 y 4 sesiones $)$ como grupales $(N=$ $52 ; \overline{\mathrm{x}}=, 75 ; \sigma_{\mathrm{X}}=, 73$; rango entre 0 y 3 sesiones).

Paralelamente al desarrollo de las sesiones de mentoría, con el propósito de identificar posibles desviaciones respecto al diseño y planificación de las mismas, los responsables del programa llevaron a cabo, el plan de seguimiento, a través del protocolo de seguimiento (instrumento utilizado para identificar posibles desviaciones que se originaran en el desarrollo de las sesiones de mentoría), mediante diferentes medidas de seguimiento (Arco y Fernández, 2007): (a) 5 sesiones grupales con los docentes-tutores del alumnado de educación obligatoria participante de cada centro educativo, con una frecuencia de 1 sesión mensual y con una duración de 1 hora cada una de ellas, con el propósito de conocer la evolución del alumnado, en términos académicos y clima escolar, (b) 5 sesiones grupales con las familias del alumnado de educación obligatoria participante de cada uno de los centros, de 1 hora de duración aproximadamente cada una de ellas y con una frecuencia de 1 sesión mensual, con el propósito de conocer la evolución del alumnado en el ambiente familiar, y (c) 4 sesiones con los mentores del alumnado de educación obligatoria participante, de carácter grupal y de 2 horas de duración, que se efectuaron una vez realizada la segunda (sesión 1 de seguimiento), la séptima (sesión 2 de seguimiento) y la duodécima sesiones de mentoría (sesión 3 de seguimiento), así como al finalizar las sesiones de mentoría (sesión 4 de seguimiento). En términos generales, las sesiones de seguimiento con los mentores, fueron registradas en los protocolos de seguimiento, donde con cada grupo de participantes valoraron las diversas actuaciones implementadas en las sesiones de mentoría y se recogieron sus percepciones respecto a la eficacia de las mismas y se les facilitó las recomendaciones oportunas para el desarrollo de las siguientes sesiones de mentoría. 
Finalmente, el plan de evaluación de resultados pretendía conocer los efectos del programa sobre el rendimiento escolar del alumnado de educación obligatoria participante y las características socio-ambientales y relaciones personales en el aula de ambas submuestras (Arco y Fernández, 2007), para lo que se tomaron medidas pretest-postest de las variables dependientes.

\section{Procedimiento y análisis de datos}

El diseño metodológico adoptado para las diferentes hipótesis fue de carácter cuasiexperimental (Ato, 2010): (a) solamente postest para la primera hipótesis, y (b) pretest-postest con un solo grupo para la segunda y tercera hipótesis.

En este sentido, y dado el carácter del objetivo propuesto, esta investigación se desarrolla en cuatro fases (Arco y Fernández, 2007): (a) análisis de necesidades, (b) diseño y planificación del programa, (c) aplicación del programa (procedimiento de selección de la muestra, plan de intervención, seguimiento y evaluación de resultados), y (d) evaluación del proceso, impacto y resultados del programa.

Por su parte, con la evaluación del proceso impacto y resultados obtenidos una vez finalizado el PHE, se pretende establecer el tipo, dirección y magnitud de los cambios producidos por la aplicación del programa en comparación con programas similares (Arco y Fernández, 2007).

Los datos fueron analizados a través del paquete estadístico 'Statistical Package for the Social Sciences' (SPSS) para Windows, versión 20.0, realizándose distribuciones de frecuencias, análisis de tablas de contingencia y coeficientes de correlación a través de los siguientes análisis estadísticos para las hipótesis planteadas: (a) primera hipótesis, estadístico de McNemar (prueba de proporciones relacionadas), y (b) segunda y tercera hipótesis, una vez comprobada la normalidad de la distribución de las puntuaciones a través de la prueba Kolmogorov-Smirnov, prueba $t$ para muestras relacionadas, prueba $z$ de los rangos con signo de Wilcoxon y valor $d$ de Cohen.

\section{Resultados}

Los resultados procedentes de la prueba de proporciones relacionadas entre el rendimiento escolar del alumnado de educación obligatoria logrado en la evaluación del primer trimestre y la evaluación del segundo y tercer trimestre del curso escolar (tablas 2 y 3), hipótesis 1, arrojan diferencias estadísticamente significativas en el área curricular de Conocimiento del medio natural, social y cultural $(p<, 05)$ (tercer trimestre), asimismo se aprecian tendencias de mejora en las áreas curriculares instrumentales (Lengua castellana y literatura, y Matemáticas) y en áreas no instrumentales (Tecnología). Además, se observa un incremento en el número de áreas curriculares con calificación de "apto", principalmente en el alumnado de EP (196 asignaturas aptas en el primer trimestre vs. 209 en el tercer trimestre), en el tercer trimestre respecto al primer trimestre. 
TABLA 2. Tabla Contingencias sobre el Rendimiento Escolar en el Primer y Segundo Trimestre del Alumnado PHE de EP y ESO

\begin{tabular}{|c|c|c|c|c|c|c|c|c|c|}
\hline \multicolumn{5}{|c|}{ Áreas Curriculares EP } & \multicolumn{5}{|c|}{ Áreas Curriculares ESO } \\
\hline \multirow[b]{2}{*}{ Trimestre 1} & \multicolumn{4}{|c|}{ Trimestre 2} & \multirow[b]{2}{*}{ Trimestre 1} & \multicolumn{4}{|c|}{ Trimestre 2} \\
\hline & Apto & No apto & Total & Ex. Sig. & & Apto & No apto & Total & Ex. Sig. \\
\hline \multicolumn{5}{|c|}{ Conocimiento del medio natural, social y cultural } & \multicolumn{5}{|c|}{ Ciencias sociales, geografía e historia } \\
\hline Apto & 21 & 3 & 24 & \multirow{3}{*}{1,00} & Apto & 20 & 5 & 25 & \multirow{3}{*}{1,00} \\
\hline No Apto & 4 & 3 & 7 & & No Apto & 4 & 9 & 13 & \\
\hline Total & 35 & 6 & 31 & & Total & 24 & 14 & 38 & \\
\hline \multicolumn{5}{|c|}{ Educación artística } & \multicolumn{5}{|c|}{ Educación plástica y visual } \\
\hline Apto & 25 & 4 & 29 & \multirow{3}{*}{0,37} & Apto & 29 & 3 & 33 & \multirow{3}{*}{0,21} \\
\hline No Apto & 1 & 1 & 2 & & No Apto & 1 & 3 & 4 & \\
\hline Total & 26 & 5 & 31 & & Total & 30 & 8 & 38 & \\
\hline \multicolumn{5}{|l|}{ Educación física } & \multicolumn{5}{|l|}{ Educación física } \\
\hline Apto & 30 & - & 30 & \multirow{3}{*}{ - } & Apto & 30 & 3 & 33 & \multirow{3}{*}{1,00} \\
\hline No Apto & 1 & - & 1 & & No Apto & 2 & 3 & 5 & \\
\hline Total & 31 & - & 31 & & Total & 32 & 6 & 38 & \\
\hline \multicolumn{5}{|c|}{ Lengua castellana y literatura } & \multicolumn{5}{|c|}{ Lengua castellana y literatura } \\
\hline Apto & 24 & - & 24 & & Apto & 23 & 2 & 25 & \\
\hline No Apto & 5 & 2 & 7 & 0,06 & No Apto & 6 & 7 & 13 & 0,28 \\
\hline Total & 29 & 2 & 31 & & Total & 29 & 9 & 38 & \\
\hline \multicolumn{5}{|c|}{ Lengua Extranjera (Inglés) } & Lengua Extranjer & nglés) & & & \\
\hline Apto & 24 & - & 24 & & Apto & 19 & 2 & 21 & \\
\hline No Apto & 5 & 2 & 7 & 1,00 & No Apto & 4 & 13 & 17 & 0,68 \\
\hline Total & 29 & 2 & 31 & & Total & 23 & 13 & 38 & \\
\hline Matemáticas & & & & & Matemáticas & & & & \\
\hline Apto & 27 & - & 27 & & Apto & 15 & 5 & 20 & \\
\hline No Apto & 2 & 2 & 4 & 0,50 & No Apto & 6 & 12 & 18 & 1,00 \\
\hline Total & 29 & 2 & 31 & & Total & 21 & 17 & 38 & \\
\hline Religión y moral & & & & & Tecnología & & & & \\
\hline Apto & 30 & 1 & 31 & & Apto & 15 & 5 & 20 & \\
\hline No Apto & - & - & - & - & No Apto & - & 8 & 8 & 0,06 \\
\hline Total & 30 & 1 & 31 & & Total & 15 & 13 & 28 & \\
\hline Educación para & ladaní & & & & Música & & & & \\
\hline Apto & 10 & - & 10 & & Apto & 24 & 5 & 29 & \\
\hline No Apto & - & - & - & - & No Apto & 3 & 6 & 9 & 0,72 \\
\hline Total & 10 & - & 10 & & Total & 27 & 11 & 38 & \\
\hline & & & & & Ciencias de la na & aleza & & & \\
\hline & & & & & Apto & 22 & 6 & 28 & \\
\hline & & & & & No Apto & 4 & 6 & 10 & 0,75 \\
\hline & & & & & Total & 26 & 12 & 38 & \\
\hline & & & & & Religión & & & & \\
\hline & & & & & Apto & 33 & - & 33 & \\
\hline & & & & & No Apto & - & - & - & - \\
\hline & & & & & Total & 33 & - & 33 & \\
\hline & & & & & $\begin{array}{c}\text { Cultura religiosa } \\
\text { Apto }\end{array}$ & 5 & - & 5 & \\
\hline & & & & & No Apto & - & - & - & - \\
\hline & & & & & Total & 5 & - & 5 & \\
\hline
\end{tabular}

Fuente: Elaboración propia 
TABLA 3. Tabla Contingencias sobre el Rendimiento Escolar en el Primer y Tercer Trimestre del Alumnado PHE de EP y ESO

\begin{tabular}{|c|c|c|c|c|c|c|c|c|c|}
\hline \multicolumn{5}{|c|}{ Áreas Curriculares EP } & \multicolumn{5}{|c|}{ Áreas Curriculares ESO } \\
\hline \multicolumn{5}{|c|}{ Trimestre 3} & \multicolumn{5}{|c|}{ Trimestre 3} \\
\hline Trimestre 1 & Apto & No apto & Total & Ex. Sig. & Trimestre 1 & Apto & No apto & Total & Ex. Sig. \\
\hline \multicolumn{5}{|c|}{ Conocimiento del medio natural, social y cultural } & \multicolumn{5}{|c|}{ Ciencias sociales, geografía e historia } \\
\hline Apto & 24 & - & 24 & \multirow{3}{*}{, $03^{*}$} & Apto & 23 & 2 & 25 & \multirow{3}{*}{1,00} \\
\hline No Apto & 6 & 1 & 7 & & No Apto & 3 & 10 & 13 & \\
\hline Total & 30 & 1 & 31 & & Total & 26 & 12 & 38 & \\
\hline \multicolumn{5}{|c|}{ Educación artística } & \multicolumn{4}{|c|}{ Educación plástica y visual } & \multirow{4}{*}{,37 } \\
\hline Apto & 28 & 1 & 29 & \multirow{3}{*}{1,00} & Apto & 30 & 4 & 34 & \\
\hline No Apto & 1 & 1 & 2 & & No Apto & 1 & 3 & 4 & \\
\hline Total & 29 & 2 & 31 & & Total & 31 & 7 & 38 & \\
\hline \multicolumn{5}{|l|}{ Educación física } & \multicolumn{4}{|l|}{ Educación física } & \multirow{4}{*}{,68 } \\
\hline Apto & 30 & - & 30 & \multirow{3}{*}{ - } & Apto & 31 & 2 & 33 & \\
\hline No Apto & 1 & - & 1 & & No Apto & 4 & 1 & 5 & \\
\hline Total & 31 & - & 31 & & Total & 35 & 3 & 38 & \\
\hline \multicolumn{5}{|c|}{ Lengua castellana y literatura } & \multicolumn{4}{|c|}{ Lengua castellana y literatura } & \multirow{4}{*}{1,00} \\
\hline Apto & 24 & - & 24 & \multirow{3}{*}{,06 } & Apto & 24 & 1 & 25 & \\
\hline No Apto & 5 & 2 & 7 & & No Apto & 2 & 11 & 13 & \\
\hline Total & 29 & 2 & 31 & & Total & 26 & 12 & 38 & \\
\hline \multicolumn{5}{|c|}{ Lengua Extranjera (Inglés) } & Lengua Extranjera & Inglés & & & \\
\hline Apto & 25 & 2 & 27 & & Apto & 19 & 2 & 21 & \\
\hline No Apto & 1 & 3 & 4 & 1,00 & No Apto & 6 & 11 & 17 & ,28 \\
\hline Total & 26 & 5 & 31 & & Total & 25 & 13 & 38 & \\
\hline Matemáticas & & & & & Matemáticas & & & & \\
\hline Apto & 27 & - & 27 & & Apto & 17 & 3 & 20 & \\
\hline No Apto & 2 & 2 & 4 &, 50 & No Apto & 7 & 11 & 18 & ,34 \\
\hline Total & 29 & 2 & 31 & & Total & 24 & 14 & 38 & \\
\hline Religión y moral & lica & & & & Tecnología & & & & \\
\hline Apto & 31 & - & 31 & & Apto & 16 & 4 & 20 & \\
\hline No Apto & - & - & - & - & No Apto & 2 & 6 & 8 & ,68 \\
\hline Total & 31 & - & 31 & & Total & 18 & 10 & 28 & \\
\hline Educación para & dadar & & & & Música & & & & \\
\hline Apto & 10 & - & 10 & & Apto & 27 & 2 & 29 & \\
\hline No Apto & - & - & - & - & No Apto & 1 & 8 & 9 & 1,00 \\
\hline Total & 10 & - & 10 & & Total & 28 & 10 & 38 & \\
\hline & & & & & Ciencias de la nat & raleza & & & \\
\hline & & & & & Apto & 24 & 4 & 28 & \\
\hline & & & & & No Apto & 3 & 7 & 10 & 1,00 \\
\hline & & & & & Total & 27 & 11 & 38 & \\
\hline & & & & & Religión & & & & \\
\hline & & & & & Apto & 33 & - & 33 & \\
\hline & & & & & No Apto & - & - & - & - \\
\hline & & & & & Total & 33 & - & 33 & \\
\hline & & & & & $\begin{array}{c}\text { Cultura religiosa } \\
\text { Apto }\end{array}$ & 5 & - & 5 & \\
\hline & & & & & No Apto & - & - & - & - \\
\hline & & & & & Total & 5 & - & 5 & \\
\hline
\end{tabular}

${ }^{\star} p<, 05 \quad$ Fuente: Elaboración propia

En cuanto a las hipótesis 2 y 3 , los resultados obtenidos de las comparaciones pretest-postest sobre las características socio-ambientales y relaciones personales en el aula para ambas submuestras (alumnado de educación obligatoria y docentes-tutores) (tablas 4 y 5), una vez comprobada la normalidad de la distribución de las puntuaciones a través de la prueba Kolmogorov-Smirnov, tanto en la fase pretest como en la fase postest, éstas no revelan diferencias estadísticamente significativas entre las puntuaciones medias obtenidas en la fase postest y pretest en las diferentes subescalas del CES (Moos et al., 1995). No obstante, estos resultados si revelan que la media de la puntuación directa conseguida por el alumnado de 
educación obligatoria en 8 de las subescalas se ha incrementado en la fase postest (Implicación, Competitividad, Organización, Claridad, Control, Afiliación, Ayuda y Tarea), y la media de la puntuación directa de los docentes-tutores en 7 de las subescalas se ha incrementado en la fase postest (Afiliación, Ayuda, Competitividad, Organización, Control, Innovación y Claridad), con respecto a la fase pretest.

Por otro lado, se ha de añadir los resultados obtenidos de los análisis del tamaño del efecto (valor $d$ de Cohen), los cuales oscilan entre -,12 y ,32 puntos en el caso del alumnado de educación obligatoria participante, y entre ,05 y ,33 puntos en el caso de los docentes-tutores participantes.

TABLA 4. Comparación Intragrupos (Prueba t) sobre Características Socio-Ambientales y Relaciones Personales en el Aula

\begin{tabular}{|c|c|c|c|c|c|c|}
\hline $\begin{array}{c}\text { Submuestra / } \\
\text { Subescala / Fase }\end{array}$ & $N$ & Media & Desviación Típica & $t$ & Sig. & $d$ \\
\hline \multicolumn{7}{|c|}{$\begin{array}{l}\text { Alumnado de EP y ESO } \\
\text { Implicación }\end{array}$} \\
\hline Pretest & 69 & 4,46 & 2,27 &,- 81 & ,42 &, 13 \\
\hline $\begin{array}{c}\text { Postest } \\
\text { Comptitividad }\end{array}$ & & 4,75 & 2,20 & & & \\
\hline $\begin{array}{l}\text { Pretest } \\
\text { Postest }\end{array}$ & 69 & $\begin{array}{l}5.83 \\
6,30\end{array}$ & $\begin{array}{l}1,76 \\
1,80\end{array}$ & $-1,63$ &, 11 &, 26 \\
\hline Organización & & & & & & \\
\hline Pretest & 69 & 4,60 & 2,07 &,- 74 & ,46 &, 13 \\
\hline $\begin{array}{l}\text { Postest } \\
\text { Claridad }\end{array}$ & & 4,87 & 2,04 & & & \\
\hline Pretest & 69 & 6,82 & 1,85 & $-1,90$ &, 06 & ,32 \\
\hline Postest & & 7,38 & 1,60 & & & \\
\hline Control & & & & & & \\
\hline $\begin{array}{l}\text { Pretest } \\
\text { Postest }\end{array}$ & 69 & $\begin{array}{l}5,74 \\
6,08\end{array}$ & $\begin{array}{l}1,71 \\
1,82\end{array}$ & $-1,20$ &, 23 & , 19 \\
\hline \multicolumn{7}{|l|}{$\begin{array}{l}\text { Docentes-tutores } \\
\text { Implicación }\end{array}$} \\
\hline $\begin{array}{l}\text { Pretest } \\
\text { Postest }\end{array}$ & 14 & $\begin{array}{l}6,85 \\
6,70\end{array}$ & $\begin{array}{l}2,41 \\
2,79\end{array}$ & ,37 & ,72 &,- 06 \\
\hline \multicolumn{7}{|l|}{ Afiliación } \\
\hline Pretest & 14 & 7,54 & 1,56 & ,00 & 1,00 & - \\
\hline \multirow{2}{*}{\multicolumn{7}{|c|}{ Ayuda }} \\
\hline & & & & & & \\
\hline $\begin{array}{l}\text { Pretest } \\
\text { Postest }\end{array}$ & 14 & $\begin{array}{l}7,92 \\
8,31\end{array}$ & $\begin{array}{l}1,50 \\
1,25\end{array}$ &,- 86 & ,41 & ,28 \\
\hline \multicolumn{7}{|l|}{ Tarea } \\
\hline Pretest & 14 & 5,92 & 2,06 & ,26 &, 80 &,- 07 \\
\hline $\begin{array}{c}\text { Postest } \\
\text { Competitividad }\end{array}$ & & 5,77 & 2,00 & & & \\
\hline Pretest & 14 & 5,10 & 1,73 &,- 58 &, 56 & ,15 \\
\hline $\begin{array}{r}\text { Postest } \\
\text { Organización }\end{array}$ & & 5,35 & 1,65 & & & \\
\hline $\begin{array}{r}\text { Pretest } \\
\text { Postest }\end{array}$ & 14 & $\begin{array}{l}6,21 \\
6,57\end{array}$ & $\begin{array}{l}2,61 \\
2,38\end{array}$ &,- 73 & ,47 & ,14 \\
\hline \multicolumn{7}{|l|}{ Control } \\
\hline Pretest & 14 & 5,31 & 1,32 &,- 25 &, 81 &, 05 \\
\hline $\begin{array}{l}\text { Postest } \\
\text { Innovación }\end{array}$ & & 5,38 & 1,39 & & & \\
\hline Pretest & 14 & 6,70 & 1,32 &,- 27 &, 80 &, 05 \\
\hline Postest & & 6,77 & 1,36 & & & \\
\hline
\end{tabular}


Tabla 5. Comparación Intragrupos (Prueba z) sobre Características Socio-Ambientales y Relaciones Personales en el Aula

\begin{tabular}{|c|c|c|c|c|c|c|}
\hline $\begin{array}{c}\text { Submuestra / } \\
\text { Subescala / Fase } \\
\end{array}$ & $N$ & Media & Desviación Típica & $z$ & Sig. & $d$ \\
\hline \multicolumn{7}{|l|}{ Alumnado de EP y ESO } \\
\hline $\begin{array}{l}\text { Pretest } \\
\text { Postest }\end{array}$ & 69 & $\begin{array}{l}6,60 \\
6,86\end{array}$ & $\begin{array}{l}2,01 \\
2,10\end{array}$ &,- 66 &, 51 & ,13 \\
\hline \multicolumn{7}{|l|}{ Ayuda } \\
\hline $\begin{array}{l}\text { Pretest } \\
\text { Postest }\end{array}$ & 69 & $\begin{array}{l}6,43 \\
6,43\end{array}$ & $\begin{array}{l}2,34 \\
2,16\end{array}$ &,- 15 & 88 & - \\
\hline \multicolumn{7}{|l|}{ Tarea } \\
\hline $\begin{array}{l}\text { Pretest } \\
\text { Postest }\end{array}$ & 69 & $\begin{array}{l}5,74 \\
6,00\end{array}$ & $\begin{array}{l}1,64 \\
1,54\end{array}$ &,- 91 & 37 & 16 \\
\hline Innovación & & & & & & \\
\hline $\begin{array}{l}\text { Pretest } \\
\text { Postest }\end{array}$ & 69 & $\begin{array}{l}5,80 \\
5,62\end{array}$ & $\begin{array}{l}1,41 \\
1,71\end{array}$ &,- 83 & ,41 &,- 12 \\
\hline \multicolumn{7}{|l|}{$\begin{array}{c}\text { Docentes-tutores } \\
\text { Claridad }\end{array}$} \\
\hline $\begin{array}{l}\text { Pretest } \\
\text { Postest }\end{array}$ & 14 & $\begin{array}{l}9,07 \\
9,62 \\
\end{array}$ & $\begin{array}{l}1,69 \\
1,63 \\
\end{array}$ &,- 65 & 10 & ,33 \\
\hline
\end{tabular}

Fuente: Elaboración propia

\section{Conclusiones}

El propósito de este trabajo de investigación fue conocer la eficacia de un programa de intervención fundamentado en las metodologías de A-S y de mentoría entre iguales, dirigido a mejorar el rendimiento escolar del alumnado de educación obligatoria participante, así como el clima social del aula, tanto desde la perspectiva del alumnado como de sus docentes-tutores. Los resultados indican una mejora leve en el rendimiento escolar del alumnado de educación obligatoria ya que hay un incremento en el número de áreas curriculares con calificación de "apto" respecto al primer trimestre del curso escolar, pero, por el contrario, no se han observado mejoras sustanciales respecto a las características socio-ambientales y relaciones personales en el aula.

En este sentido, la participación en el PHE no ha tenido todo el efecto esperado sobre el rendimiento escolar del alumnado de educación obligatoria, a diferencia de los resultados obtenidos por otros estudios comparables como por ejemplo Westover (2012). No obstante, si se realiza un análisis más exhaustivo, caso a caso, se aprecian tendencias de mejora hasta en un $55 \%$ de los componentes de la submuestra, sobre todo en aquellos que desarrollaron más de 14 sesiones de mentoría, y principalmente en las áreas instrumentales. Estos resultados se alinean también con los encontrados por Scales, Blyth, Berkas, y Kielsmeier (2000), y al igual que estos autores entendemos que la falta de efectos en mayor medida significativos sobre la variable dependiente éxito escolar pueden estar ligados a las dificultades o limitaciones del tipo de diseño de investigación utilizado, es decir, medidas pretest-postest en un solo grupo para detectar el efecto del tratamiento debido, por ejemplo a las diferencias en contenidos entre las diferentes evaluaciones, las diferentes formas de evaluar de los docentes, o el carácter multicausal de la variable dependiente, etc. En caso de ser cierta la hipótesis, estaríamos ante un error frecuente en estudios de investigación educativa considerada como "Error tipo II" que afecta a la validez de las 
conclusiones estadísticas, y que consiste en no detectar el efecto de la intervención cuando existe realmente (Shadish, Cook, y Campbell, 2002).

Por otro lado, en cuanto a la ausencia de efectos estadísticamente significativos para ambas submuestras en la variable características socio-ambientales y relaciones personales en el aula, las recomendaciones de expertos en metodología y análisis estadísticos apuntan que los resultados de este tipo de investigaciones deben interpretarse también en función del tamaño del efecto, de cara a identificar su significatividad práctica (Cohen 1988; Ledesma, Macbeth, y Cortada 2008). En este sentido, entre los resultados más significativos del tamaño del efecto destacan, en el caso del alumnado de educación obligatoria, las subescalas de Claridad $(d=, 32)$ y Competitividad $(d=, 26)$, mientras que en el caso de los docentes-tutores destacan las subescalas de Claridad $(d=, 33)$ y Ayuda $(d=, 28)$, que muestran que un miembro hipotético de ambas submuestras puede alcanzar puntuaciones superiores al $58 \%$ en la fase postest respecto a la fase pretest. En el resto de subescalas, los resultados del tamaño del efecto no han sido muy elevados (con valores de entre ,12 y ,33), pero como plantea Coe (2002), un cambio superior a ,1 puntos se puede considerar un incremento bastante significativo, sobre todo si se puede apreciar de manera uniforme en todos los miembros de las submuestras, como sucede en este trabajo.

Efectivamente, se puede concluir que la participación en el PHE mediante la metodología de A-S y la mentoría entre iguales ha generado ciertos cambios significativos en la cantidad y calidad de las interacciones del alumnado de educación obligatoria participante y, por tanto, una optimización del clima social, lo cual, a su vez se suele relacionar con un incremento del éxito o rendimiento escolar del alumnado (Hadre, Crowson, Debacker, y White, 2007) y el bienestar emocional del alumnado (Reddy, Rhodes, y Mulhall, 2003; Ros, Filella, Ribes, y Pérez, 2017). Al igual que Cunningham (2002) consideramos que la cadena de cambios observada en los comportamientos del alumnado participante tales como mejorar su interés por las tareas escolares, incrementar su nivel de participación en ellas, aumentar su esfuerzo por obtener calificaciones elevadas, mejorar la organización de sus tareas y planificación de actividades, optimizar sus relaciones de amistad, preocupación, ayudar y/o apoyar a los otros (Estévez, Murqui, Musiti, y Moreno, 2008; McMahon, Wernsman, y Rose, 2009; Prado, Ramírez, y Ortiz, 2010; Ros et al., 2017). Condiciona favorablemente el comportamiento de los docentes (Alonso, 2007), en aspectos esenciales tales como el seguimiento de sus instrucciones, respetar las normas, prestarles atención, ignorar distracciones y no molestar (Cooperación) (Mudarra y GarcíaSalguero, 2016).

Desde el punto de vista de las limitaciones que presenta este estudio y en la línea de lo que otros autores plantean en programas similares (Amaral y Vala, 2009; Goff, 2011; Hu y Ma, 2010; O'Brien, Llamas, y Stevens, 2012; Permaul, 2009; Scales et al., 2000; Westover, 2012; Wilczenski y Coomey, 2007), para reducir la probabilidad de error tipo II, que comentábamos anteriormente, así como para mejorar la potencia del programa, se podrían introducir los siguientes cambios: (a) utilizar diseños experimentales o en su defecto cuasi-experimentales con grupo control de manera que se puedan evitar las dificultades ligadas al actual diseño, (b) aumentar el $\mathrm{N}$ de la submuestra de docentes-tutores y por tanto la variabilidad y posibilidad de detectar los efectos buscados, (c) hacer coincidir el inicio del programa con el inicio del curso con lo cual no sólo aumentamos la duración del programa, y por tanto, la exposición al mismo, sino que también quizás prevenimos la aparición temprana de diferencias en rendimiento más difíciles de atender desde el programa planificado, y (d) las variaciones en cuanto al número de sesiones de mentoría realizadas (rango de entre 9 y 18 sesiones) revelan efectos en los resultados obtenidos con lo que introducir mecanismos que prevengan estas diferencias es fundamental. Desde nuestra experiencia entendemos que la superación total o parcial de estas limitaciones pasa necesariamente por incrementar el compromiso de los docentes participantes y el departamento de orientación, asignándoles un papel protagonista en la detección de necesidades, puesta en marcha y evaluación del programa, así como el acompañamiento al alumnado participante. De esta forma conseguiremos reforzar el principio de intervención social en orientación tal y como apuntan 
Gezuraga y Malik (2015) explorando de manera más proactiva los valores, intereses, capacidades, etc., de los participantes y las necesidades y demandas sociales.

En definitiva, esta investigación ha permitido conocer algunos de los beneficios, más allá de la satisfacción personal, que se derivan de la participación en un programa de intervención dirigido a la prevención del fracaso y abandono escolar temprano, y resalta la importancia de establecer un cambio de filosofía y una visión compartida a la hora de abordar este fenómeno (Edwards, 2002). No obstante, es necesario continuar diseñando, planificando, aplicando y evaluando intervenciones, con el mayor grado de experimentalidad posible, salvando las restricciones éticas (ej., un grupo control puede implicar un uso ineficiente de los recursos humanos), que permitan hacer frente a este fenómeno extremadamente complejo como es el fracaso y abandono escolar temprano.

\section{Referencias bibliográficas}

Alonso, P. (2007). Evaluación formativa y su repercusión en el clima del aula. Revista de Investigación Educativa, 25(2), 389-402.

Amaral, K.E. y Vala, M. (2009). What teaching teaches: Mentoring and the performance gains of mentors. Journal of Chemical Education, 86, 630-633. doi:10.1021/ ed086p630.

Arco, J.L. Y Fernández, F.D. (2007). Evaluación de programas en educación no formal. En C. Torres y J.A. Pareja (Coords.), La educación no formal y diferenciada. Fundamentos didácticos y organizativos (667-690). Madrid: Editorial CCS.

Arco, J.L. Fernández, F.D. y Fernández, J.M. (2011). The impact of a peer-tutoring program on quality standars in higher education. Higher Education, 62(6), 773-788. doi:10.1007/s10734011-9419-x

Ato, M. (2010). Tipología de diseños cuasiexperimentales. En M.T. Anguera, J. Arnau, M. Ato, T. Martínez, J. Pascual y G. Vallejo (Eds.), Métodos de investigación en Psicología (245-269). Madrid: Síntesis.

Boland, J. (2010). Teaching and learning through civic engagement: Prospects for sustainability in teacher education. Issues in Educational Research, 20(1), 1-20.

Coe, R. (2002). It's the effect size, stupid. What effect size is and why it is important. British Educational Research Association Annual Conference. United Kingdom, Exeter.

Cohen, J. (1988). Statistical power analysis for the behavioral sciences (2 $2^{\underline{a}}$ ed.). Hillsdale, NJ: Lawrence Earlbaum Associates.

Cunningham, E.G. (2002). Developing a measurement model for coping research in early adolescence. Educational and Psychological Measurement, 62, 147-163. doi:10.1177/0013164402062001010

Edwards, S. (2002). Creating the climate for positive school change. En D. Rea y J.J. Bergin (Eds.), Safeguarding our youth: Successful school \& community programs (27-33). New York, NY: McGraw-Hill.

Estévez, E., Murgui, S., Musitu, G. Y Moreno, D. (2008). Clima familiar, clima escolar y satisfacción con la vida en adolescentes. Revista Mexicana de Psicología, 25(1), 119-128.

Gezuraga, M. Y Malik, B. (2015). Orientación y acción tutorial en la Universidad: Aportes desde el Aprendizaje-Servicio. Revista Española de Orientación y Psicopedagogía, 26(2), 8-25. 
Goff, L. (2011). Evaluating the outcomes of a peer-mentoring program for students transitioning to postsecondary education. Canadian Journal for the Scholarship of Teaching and Learning, 2(2), 1-12. doi:10.5206/cjostl_rcacea.2011.2.2

González, L., Rivera, E. Y Trigueros, C. (2014). La interacción social en el contexto del aula de Educación Física. Revista de currículum y formación del profesorado, 18(2), 305-320.

Hadre, P.L., Crowson, M.H., Debacker, T.K. Y White, D. (2007). Predicting the academic motivation of rural high school students. Journal of Experimental Education, 75(4), 247-269. doi:10.3200/JEXE.75.4.247-269

Hervás, M., Fernández, F.D., Arco, J.L. Y Miñaca, M.I. (2017). Efectos de un programa de Aprendizaje-Servicio en el alumnado universitario. Electronic Journal of Research in Educational Psychology, 15(41), 126-146. doi:http://dx.doi.org/10.14204/ejrep.41.16049

$\mathrm{Hu}, \mathrm{S}$. Y Ma, Y. (2010). Mentoring and student persistence in college: A study of the Washington State Achievers Program. Innovative Higher Education, 35, 329-341. doi:10.1007/s10755-0109147-7

Knapp, T., Fisher, B. Y Levesque-Bristol, C. (2010). Service-Learning's impact on college students' commitment to future civic engagement, self efficacy, and social empowerment. Journal of Community Practice, 18, 233-251. doi:10.1080/10705422.2010.490152

Lara, B., González, A., González, M.A. Y Martínez, M.G. (2014). Fracaso Escolar: conceptualización y perspectivas de estudio. Revista de Educación y Desarrollo, 30, 71-83.

Ledesma, R., Macbeth, G. Y Cortada, N. (2008). Tamaño del efecto: Revisión teórica y aplicaciones con el sistema estadístico ViSta. Revista Latinoamericana de Psicología, 40(3), 425-439.

Lord, J., Mourtzanos, E., Mclaren, K., Murray, S., Kimmel, R. Y Cowley, D. (2012). A peer mentoring group for junior clinician educators: Four years' experience. Academic Medicine, 87(3), 378-383. doi:10.1097/ACM.0b013e3182441615

Maloney, M. Y Griffith. K. (2013). Occupational therapy students' development of therapeutic communication skills during a service-learning experience. Occupational Therapy in Mental Health, 29, 10-26. doi:10.1080/0164212X.2013.760288

Maloney, M., Myers, C. Y Bazyk, J. (2014). The influence of a Community-Based Service-Learning experience on the development of Occupational Therapy students' feelings of civic responsibility. Occupational Therapy in Mental Health, 30(2), 144-161. doi:10.1080/0164212X.2014.910160

Martínez, V. (2009). Investigación y reflexión sobre condicionantes del fracaso escolar. Revista Latinoamericana de Estudios Educativos, 39(1-2), 11-38.

Martínez, R. (2010). El método de encuestas por muestreo: Conceptos básicos. En M.T. Anguera, J. Arnau, M. Ato, R. Martínez, J. Pascual y G. Vallejo (Eds.), Métodos de investigación en Psicología (385-43). Madrid: Síntesis.

Mcintyre, K.A. y Sellnow, D.D. (2014). A little bit can go a long way: An examination of required service in the basic communication course. Communication Teacher, 28(1), 57-73. doi:10.1080/17404622.2013.843012

Mcmahon, S.D., Wernsman, J. y Rose, D. (2009). The relation of classroom environment and school belonging to academic self-efficacy among urban fourth- and fifth- grade students. The Elementary School Journal, 109, 267-281. doi:10.1086/592307

Miñaca, M.I. y Hervás, M. (2013). Intervenciones dirigidas a la prevención del fracaso y abandono escolar. Un estudio de revisión. Revista de Educación Comparada, 21, 203-220. 
Molina, N. y Pérez, I. (2006). El clima de relaciones interpersonales en el aula un caso de estudio. Paradigma, 27(2), 193-219.

Moos, R.H. y Trickett, E.J. (1974). Classroom Environment Scale Manual. Palo Alto: Consulting Psychologists Press.

Moos, R.H., Moos, B.S. y Trickett, E.J. (1995). Escala de Clima Social: Centro Educativo (CES). Madrid: TEA Ediciones.

Mudarra, M. y García-Salguero, B. (2016). Habilidades sociales y éxito académico: Expectativas de los profesores de Educación Secundaria. Revista Española de Orientación y Psicopedagogía, 27(1), 114-133.

Noam, G. y Fiore, N. (2004). Relationships across multiple setting. An Overview. New Direction for Youth Development, 103, 9-16. doi:10.1002/yd.87

O'Brien, M., Llamas, M. y Stevens, E. (2012). Lessons learned from four years of peer mentoring in a tiered group program within education. Journal of the Australian \& New Zealand Student Services Association, 40, 7-15.

Permaul, J.S. (2009). Theoretical bases for service-learning: Implications for program design and effectiveness. New Horizons in Education, 57(3), 1-7.

Prado, V.M., Ramírez, M.L. y Ortiz, M.S. (2010). Adaptación y validación de la escala de clima social escolar (CES). Actualidades Investigativas en Educación, 10(2), 1-13. doi:http://dx.doi.org/10.15517/aie.v10i2.10121

Pulido, F. y Herrera, F. (2016). El miedo como predictor del rendimiento académico: El contexto pluricultural de Ceuta. Revista Española de Orientación y Psicopedagogía, 27(2), 29-44.

Reddy, R., Rhodes, J.E. y Mulhall, P. (2003). The influence of teacher support on student adjustment in the middle school years: A latent growth curve study. Development and Psychopathology, 15, 119-138. doi:http://dx.doi.org/10.1017.S0954579403000075

Risquez, A. (2011). Peer electronic mentoring for transition into university: A theoretical review. Revista Española de Orientación y Psicopedagogía, 22(3), 232-239.

Ros, A., Filella, G., Ribes, R. y Pérez, N. (2017). Análisis de la relación entre competencias emocionales, autoestima, clima del aula, rendimiento académico y nivel de bienestar en educación primaria. Revista Española de Orientación y Psicopedagogía, 28(1), 8-18.

Sammons, P. y Bakkum, L. (2011). Effective schools, equity and teacher effectiveness: A review to the literature. Revista de Currículum y Formación del Profesorado, 15(3), 9-26.

Scales, P., Blyth, D., Berkas, T. y Kielsmeier, J. (2000). The effects of service-learning on middle school students' social responsibility and academic success, Journal of Early Adolescence, 20, 331-358. doi:10.1177/0272431600020003004

Shadish, W., Cook, T. y Campbell, D. (2002). Experimental and quasi-experimental designs for generalized causal inference. Boston, MA: Houghton Mifflin.

Stanton, T. (2014). Practice makes perfect: Classrooms and curricula that generate civic minds and actions. The Journal of General Education, 63(1), 35-46. doi:10.1353/jge.2014.0001

Vaknin, L.W. y Bresciani, M. (2013). Implementing quality Service-Learning Programs in Community Colleges. Community College Journal of Research and Practice, 37(12), 979-989. doi:10.1080/10668926.2010.515515

Weiler, D., Lagoy, A., Crane, E. y Rovner, A. (1998). An evaluation of K-12 service-learning in California: Phase II final report. Emeryville, CA: RPP International with the Search Institute. 
Westover, J. H. (2012). Academic service-learning across disciplines. Models, outcomes and assessment. Champaign, IL: Common Groum Publishing.

Wilczenski, F.L. y Coomey, S.M. (2007). A practical guide to service learning: Strategies for positive development in schools. New York, NY: Springer.

\section{Fuentes electrónicas}

Consejo Escolar Del Estado. (2015). Informe 2015 sobre el estado del sistema educativo. Madrid: Ministerio de Educación, Cultura y Deporte. Recuperado el 15 de septiembre de 2016, de http://ntic.educacion.es/cee/informe2015/i2015cee.pdf

Fernández, M., Mena L. Y Riviere, J. (2010). Fracaso y abandono escolar España. Barcelona: Colección de Estudios Sociales 'la Caixa'. Recuperado el 22 de mayor de 2013, de https://obrasocial.lacaixa.es/deployedfiles/obrasocial/Estaticos/pdf/Estudios_sociales/vol29_co mpleto_es.pdf

Instituto Nacional De Evaluación Educativa. (2014). Sistema estatal de indicadores de la educación 2014. Madrid: Ministerio de Educación, Cultura y Deporte. Recuperado el 13 de junio de 2015, de http://www.mecd.gob.es/dctm/inee/indicadores-educativos/seie2014/seie2014-libro-completo-4062014-f.pdf?documentld=0901e72b8197c344

Instituto Nacional de Evaluación Educativa. (2015). Sistema estatal de indicadores de la Educación 2015. Madrid: Ministerio de Educación, Cultura y Deporte. Recuperado el 23 de Octubre de 2016, de http://www.mecd.gob.es/servicios-al-ciudadano$\mathrm{mecd} / \mathrm{dms} / \mathrm{mecd} / \mathrm{servicios}$-al-ciudadano-mecd/estadisticas/educacion/indicadorespublicaciones-sintesis/sistema-estatal-indicadores/SEIE 2015.pdf

Instituto Nacional de Evaluación Educativa. (2016). Sistema estatal de indicadores de la Educación 2016. Madrid: Ministerio de Educación, Cultura y Deporte. Recuperado el 17 de Noviembre de 2016, http://www.mecd.gob.es/dctm/inee/indicadores/2016/seie2016okkk.pdf?documentld=0901e72 $\underline{\mathrm{b} 8222 \mathrm{f} 7 \mathrm{~d} 1}$

MENTOR/National Mentoring Partnership. (2005). Cómo construir un programa de mentoría exitoso utilizando los elementos de la práctica efectiva. Alexandria, VI: A Mentor/National Mentoring Partnership. Recuperado el 11 de enero de 2009, de http://docplayer.es/1565837Como-construir-un-programa-de-mentoria-exitoso.html

Fecha de entrada: 29 Octubre 2016

Fecha de revisión: 13 Marzo 2018

Fecha de aceptación: 20 Marzo 2018 